\title{
Rapid learning of complex sequences with time constraints: A dynamic neural field model
}

\author{
Flora Ferreira, Weronika Wojtak, Emanuel Sousa, Luís Louro, Estela Bicho, and Wolfram Erlhagen
}

\begin{abstract}
Many of our sequential activities require that behaviors must be both precisely timed and put in the proper order. This paper presents a neuro-computational model based on the theoretical framework of Dynamic Neural Fields that supports the rapid learning and flexible adaptation of coupled order-timing representations of sequential events. A key assumption is that elapsed time is encoded in the monotonic buildup of self-stabilized neural population activity representing event memory. A stable activation gradient over subpopulations carries the information of an entire sequence. With robotics applications in mind, we test the model in simulations of a learning by observation paradigm, in which the cognitive agent first memorizes the order and relative timing of observed events and, subsequently, recalls the information from memory taking potential speed constraints into account. Model robustness is tested by systematically varying sequence complexity along the temporal and the ordinal dimension. Furthermore, an adaptation rule is proposed that allows the agent to adjust in a single trial a learned timing pattern to a changing temporal context. The simulation results are discussed with respect to our goal to endow autonomous robots with the capacity to efficiently learn complex sequences with time constraints, supporting more natural human-robot interactions.
\end{abstract}

Index Terms - sequence learning, interval timing, dynamic field theory, neurocomputational model, human-robot interactions.

\section{INTRODUCTION}

The ability to acquire sensitivity about ordinal and temporal regularities in sequential activities is fundamental to our adaptive behavior in an inherently dynamic environment [1]. We can use knowledge of these regularities to predict what sensory or motor event comes next and when it will happen. The benefits of predictive in contrast to pure reactive processing of events have been widely recognized both in the perceptual and the motor domain [2]. It allows the cognitive system to optimize behavior and, thus, save precious resources. Fluent and skillful performance of many of our everyday sequential behaviors requires that the coupling of the internally guided processing of the order, interval, and duration of events is rather tight. This is immediately evident for tasks like tapping your fingers to the rhythm of a tune, but it also applies to common sequential actions like starting the car when the traffic light turns green, or smoothly handing over a series of objects to another person. The learning mechanisms responsible for forming such coupled order-timing representations and their putative neural substrate are a matter of considerable debate [3], [4], [5], [6], [7]. Valuable insight comes from experiments with the serial reaction time (SRT) paradigm developed by

F. Ferreira, W. Wojtak, and W. Erlhagen are with Center of Mathematics, University of Minho, Portugal e-mail: wolfram.erlhagen@math.uminho.pt.

W. Wojtak, E. Sousa, L. Louro and E. Bicho are with Center Algoritmi, University of Minho, Portugal.
Nissen and Bullemer [8], which has been extensively used in the past to study the cognitive and neural mechanisms supporting perceptual and motor sequence learning. In the basic form of this paradigm, participants are instructed to react as quickly and as accurately as possible to successively presented stimuli by pressing the assigned response keys. The sequence of targets follows a fixed order which repeats many times. Sequence-specific learning is shown by being faster and more accurate on blocks with patterned trials as opposed to blocks of randomly ordered stimuli. To access temporal learning, the time intervals between successive stimuli can be also manipulated, following a structured or a random pattern. The best performance is achieved when the temporal and the ordinal sequences are correlated and a predictive relationship between action order and timing exists. This finding has been interpreted as evidence that sequential and temporal information are bounded together in memory during learning ([6], [7], but see [5]). SRT learning bears some similarity with learning to play a melody on a piano, by pressing the right keys in the correct order and time in response to sensory cues [9]. Different to the temporal goal of an SRT paradigm, which does not require a precise temporal expectation of upcoming events, musical performance requires that timing becomes fully integrated into the procedural representation since the same series of pitch events may have a different meaning depending on the precise temporal intervals separating them [10].

In this paper, we present a computational model for learning of timed sequential events based on the theoretical framework of Dynamic Neural Fields (DNF) [11], [12]. It implements neuro-plausible processing mechanisms supporting the efficient acquisition and flexible reproduction of complex sequences with strong time constraints. The model is an extension of a previous DNF model that we have applied to endow autonomous robots with the capacity to learn the serial order of sequential tasks by observation [13], [14], [15]. The most significant advances compared to the previous work are in the processing of temporal characteristics of sequential events and a systematic test of model robustness. The lack of a time processing ability in most current robotic cognitive systems is considered a major obstruction to the seamless integration of robots into human environments [16]. The DNF model is able to memorize and recall not only the order and relative timing of events but also their durations. Furthermore, we propose a fast adaptation rule that allows a cognitive agent to synchronize the internally guided timing of the entire sequence or individual events with external cues and to correct timing errors. Such temporal flexibility must be built into 
any robotics system that is supposed to operate autonomously in human environments (e.g., human-robot interactions [17], [18]). Crucial for future real-world robot implementations, we test the robustness of the model performance by analyzing the impact of different noise sources on the occurrence of errors in serial order and on the precision of event timing.

The dynamic neural field model implements several key processing mechanisms which are commonly believed to support the accumulation, storage, and recall of sensory events in associative brain areas. The short-term memory of an event is represented through the persistent activity of a neural population tuned to the stimulus dimension guiding sequence learning. Dynamic field theory explains a self-sustained activity pattern that outlasts the presence of a brief input by strong recurrent excitatory and inhibitory interactions within neural populations [19], [20]. We propose a separate threshold accommodation dynamics to account for the finding that the input-dependent persistent activity is not static but often varies systematically over the course of maintenance. The observed monotonic increase (or decrease) has been interpreted as evidence that the self-sustained population activity encodes in addition to input identity also elapsed time [21], [22], [23]. As a result, the neural dynamics of a "memory field" establishes in response to a series of sensory cues a gradient of persistent activity over subpopulations which carries the information about the sequential order and the relative timing of events. The combined information about the two task variables is sequentially recalled in a "decision field" which receives the stable activation pattern as subthreshold input. All subpopulations encoding specific events thus appear to be preactivated with a strength reflecting their rank order [24], [25], [26]. Following the framework of a bounded accumulation of stochastic evidence frequently applied in biological models of sensorimotor decisions ([27], see also [28]), a continuous rise of population activity from the pre-activated state to a pre-specified threshold defines the expected event timing. A context-dependent, highly flexible timing of the entire sequence is achieved without recourse to any changes in the network structure by adapting the initial condition or the input to the ramp-to-threshold dynamics [29].

The remainder of the paper is organized as follows: in Section II, we present an overview of the DNF model and describe its mathematical implementation. In Section III, we illustrate how the fast learning mechanism encodes order-timing information of sequential events including repetitions. Next, in Section IV, it is shown how the stored information can be recalled with different execution speeds, preserving relative event timing. Section $\mathrm{V}$ presents two important model extensions, the encoding of event duration and the synchronization with external reference cues signaling an expected timing pattern. Sequence errors and the precision of time measurements are addressed in Section VI using a stochastic version of the DNF model. In section VII, a critical discussion of the results is provided, and finally, in Section VIII, conclusions and future work are presented.

\section{OVERVIEW OF THE DNF MODEL}

Dynamic neural fields provide a rigorous theoretical framework to analyze and implement neural computations that bridge gaps between sensation and action in order to mediate working memory, action preparation, and decision making (for review see [11]). DNFs are defined over continuous perceptual or motor variables such as orientation, color, spatial object location or movement direction. A DNF describes the activation dynamics of a recurrently connected neural population tuned to the continuous dimension [20]. Crucial for the present sequence learning experiment, the field dynamics supports the existence of localized activity patterns which are initially triggered by transient sensory signals and/or input from connected populations but become self-sustained due to strong excitatory and inhibitory interactions within the population. The steady states of the field dynamics are often called bump attractors since they represent bell-shaped activity patterns in metric space. The translation-invariant connection structure enables a DNF to hold a continuous family of such attractor states [30]. The bump location thus provides a substrate for encoding continuous variables.

To understand the model architecture and the implemented neural computations depicted in Fig. 1, it is helpful to think about a concrete robotics experiment. It is motivated by our previous research on natural human-robot collaboration in an assembly paradigm [17], [31], [18]. A robot learns by observation the serial order and relative timing of object handovers between an assistant and an operator to subsequently substitute the assistant in the joint task. Sequence learning is guided by the object feature color. Additional start and stop cues define sequence duration. An important prerequisite is that robot learning should be fast and efficient since the human tutors cannot be expected to repeat the demonstration many times.

When the robot camera detects that the assistant grasps an object with a specific color for handing it over, the camera input creates a bump attractor in the perceptual field, $u_{P_{o n}}$, defined over this feature dimension. The bump position represents the hue value in color space coordinates. Four different color events are used in the model simulations ( $\mathrm{R}=\mathrm{red}, \mathrm{G}=$ green, $\mathrm{B}=$ blue, $\mathrm{M}=$ magenta). A bump in the perceptual field $u_{P_{o n}}$ drives through excitatory connections (solid line) the evolution of a localized activity pattern at the corresponding site in the "on" sequence memory field $u_{M_{o n}}$. We use here the prefix "on" to distinguish the memory of cue onset from the memory of cue offset that we exploit in a model extension to represent also event duration (see section V-B). Inhibitory feedback (dotted line) from $u_{M_{o n}}$ to $u_{P_{o n}}$ destabilizes the existing bump in the perceptual field. This ensures that newly arrived localized input to $u_{P_{o n}}$ will automatically drive the evolution of a bump at a different field location even if the specific cue value is repeated during the course of the sequence. A demonstrated series of color events creates a multi-bump pattern in $u_{M_{o n}}$ that stores all sequence elements with a strength of activation decreasing from element to element as a function of elapsed time since sequence onset. Persistent population activity, which slowly increases as a function of elapsed 
time, is achieved by a threshold accommodation dynamics. To guarantee robustness of the encoding process in the face of noisy and potentially incomplete sensory information, a fading memory trace of the multi-bump in $u_{M T}$ builds up during successive demonstrations. Subpopulations in $u_{M T}$ get excitatory input from corresponding populations in $u_{M_{o n}}$ and project via excitatory connections to the associated neural pools in $u_{P_{o n}}$. The resulting preshaping of neural populations in $u_{P_{o n}}$ based on prior experience modulates perceptual thresholds and speeds up the processing of inputs [32].

The three DNFs on bottom of Fig. 1 become active during sequence recall. The "on decision" field $u_{D_{o n}}$ receives the multi-bump pattern storing the order and relative timing of events in $u_{M_{o n}}$ as subthreshold input. A bump in the "sequence start" field $u_{\text {Start }}$, which is initially triggered by the presentation of the start cue, initiates a continuous rise of the baseline activity in $u_{D_{o n}}$. This results in a parallel ramping activity of all pre-activated subpopulations until the threshold for creating a bump is reached, starting with the population with the highest pre-activation and ending with the population with the lowest pre-activation. In robotics applications, we use the instant when a specific subpopulation in $u_{D_{o n}}$ reaches the threshold to initiate a motor response associated with this population [13], [14]. In the object transfer task for instance, the robotics assistant would start the grasping of the object with a specific color to hand it over to the operator. The excitatory-inhibitory connections between associated populations in $u_{D_{o n}}$ and the working memory field $u_{W M}$ guarantee that the suprathreshold activity representing the latest recall decision becomes first stored in $u_{W M}$ and subsequently suppressed in $u_{D_{o n}}$. If the feedback inhibition works properly, potential repetition errors during sequence recall are excluded.

\section{A. Implementation details}

In the following, we present the basic model equations that implement the neural processing mechanisms just described (for a full model description and parameter values see the Appendices A and B). The population dynamics in each field is governed by a field equation first proposed and analyzed by Amari [19]:

$$
\begin{aligned}
& \tau \frac{\partial u(x, t)}{\partial t}=-u(x, t)+\int w(|x-y|) f(u(y, t)) \mathrm{d} y \\
& +S(x, t)-h+c_{u} \xi(x, t)
\end{aligned}
$$

where $u(x, t)$ represents the activity at time $t$ of a neuron tuned to the feature value $x$. The global inhibition, $h>0$, determines the baseline level to which field excitation decays without external stimulation. $S(x, t)$ represents time dependent localized input at site $x$ from the vision system and/or connected fields. The constant $\tau>0$ defines the time scale of the field dynamics. The additive, spatially correlated noise $\xi(x, t)$ with strength $c_{u}>0$ is used in stochastic model simulations. The firing rate function $f(u)$ is taken as the Heaviside step function with threshold 0 . The interaction kernel $w(|x-y|)$ defines the coupling strength between any two neurons in the field which is assumed to depend on Euclidian distance. For fields where at most one bump should evolve at any time (e.g.,

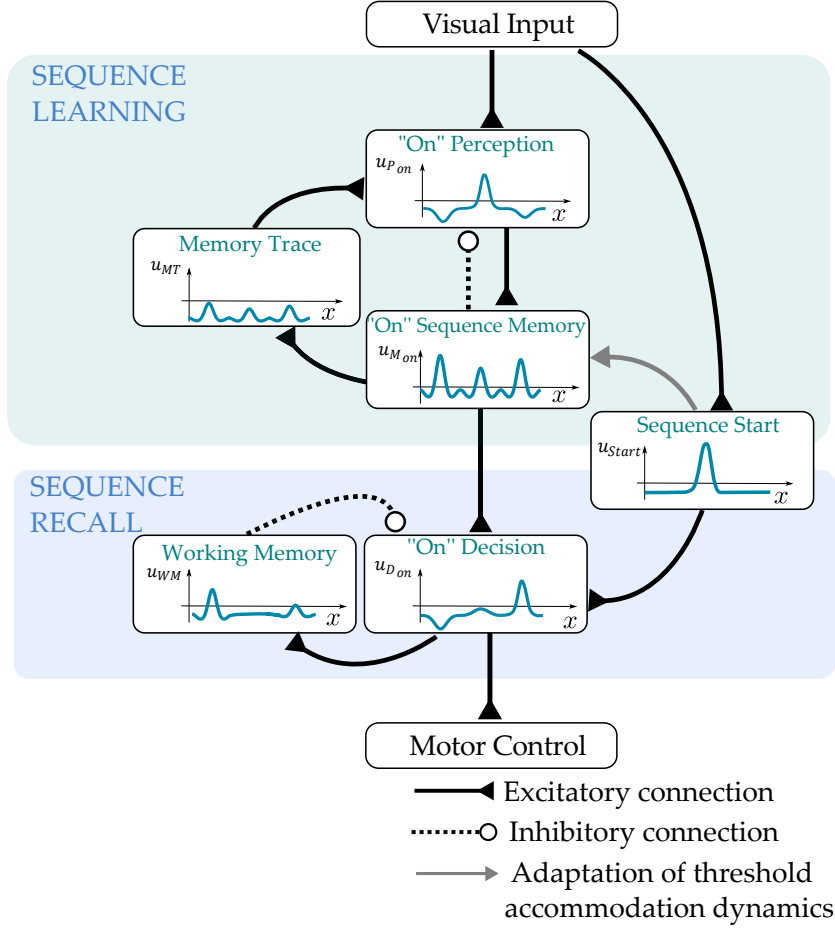

Fig. 1. Schematic view of the DNF architecture with several interconnected fields implementing sequence learning and sequence recall. Dotted lines represent inhibitory connections, solid lines excitatory connections. The arrow from the sequence start field $u_{S t a r t}$ to the memory field $u_{M_{o n}}$ indicates that the time window for the threshold accommodation dynamics in $u_{M_{o n}}$ is controlled by the presence of a bump in $u_{\text {Start }}$. For details see the text.

$\left.u_{D_{o n}}, u_{P_{o n}}\right)$, we use a standard kernel of lateral inhibition type [19]:

$$
w_{l a t}(x)=w_{e x c} e^{\left(-\frac{x^{2}}{2 \sigma_{e x c}^{2}}\right)}-w_{i n h},
$$

where $w_{\text {exc }}>0$ and $\sigma_{\text {exc }}>0$ define, respectively, the amplitude and standard deviation, and $w_{i n h}>0$ defines the approximately constant inhibition for distant neurons. To enable input driven, multi-bump solutions in the memory fields, we adopt a kernel with oscillatory rather than monotonic decay [33], [34]:

$$
w_{o s c}(x)=A e^{-b|x|}(b \sin |\alpha x|+\cos (\alpha x)),
$$

where $b>0$ determines the rate at which the oscillations of $w$ decay with distance, and $A>0$ and $0<\alpha \leq 1$ control the amplitude and the spatial phase of $w$, respectively. To establish a stable activation gradient in $u_{D_{o n}}$ representing the order and relative timing of perceived events, we chose the baseline activity to be time dependent, $h=h(t)$. Note that by including $h(t)$ in the definition of the firing rate function, $f(u, h)=$ $f(u-h)$, it becomes clear that changing the baseline level in the field equation is functionally equivalent to changing the threshold of the transfer function $f(u)$. Following the idea of a phenomenological model of threshold accommodation in dynamic fields [35], we apply the following state-dependent dynamics for $h_{M_{o n}}$ :

$$
\begin{array}{r}
\frac{\partial h_{M_{o n}}(x, t)}{\partial t}=\beta_{M} f\left(u_{M_{o n}}(x, t)\right) \int_{\Omega} f\left(u_{\text {Start }}(x)\right) \mathrm{d} x \\
+\left[1-f\left(u_{M_{o n}}(x, t)\right)\right]\left[-h_{M_{o n}}(x, t)+h_{M_{0}}\right]
\end{array}
$$


where $f(u)$ is again the step function, $h_{M_{0}}<0$ defines the baseline activation to which $h_{M_{o n}}$ converges without suprathreshold activity at position $x$, and $\beta_{M}>0$ sets the buildup rate of activity at field sites that have received localized input. The time window for the buildup is controlled by the presence of a stable bump in $u_{\text {Start }}$ which is initially triggered by the presentation of the sequence start signal at $t_{\text {start }}=0$ (e.g., an additional color cue in the robotics application). The integral over the interval with suprathreshold activity, $\Omega=\left\{x: u_{\text {Start }}(x) \geq 0\right\}$, is simply the bump width, $\int_{\Omega} f\left(u_{\text {Start }}(x)\right) d x=a>0$. The threshold accommodation dynamics ends with the presentation of the stop signal at $t=t_{\text {end }}$. This signal is modeled as a transient inhibitory input to $u_{\text {start }}$ which destabilizes the bump. The suprathreshold activity relaxes to the subthreshold baseline state, that is, $\int_{\Omega} f\left(u_{\text {Start }}(x)\right) d x=0$.

To directly compare during sequence recall the predicted with the demonstrated timing pattern we further assume a common start signal at time $t_{\text {start }}=0$ for sequence demonstration and production. The stable bump in $u_{\text {Start }}$ initiates the autonomous sequence recall in the "on decision" field $u_{D_{o n}}$. The ramp-to threshold dynamics of the baseline activity is governed by the equation:

$$
\frac{\mathrm{d} h_{D}(t)}{\mathrm{d} t}=\beta_{D} \int_{\Omega} f\left(u_{\text {Start }}(x)\right) \mathrm{d} x+c_{h} \epsilon(t)
$$

where $\beta_{D}$ is a scaling parameter to control the speed of sequence recall (see section IV). Random fluctuations in the ramping dynamics, which we apply to address the precision of timing (see section VI-B), are modeled by Gaussian noise, $\epsilon(t)$, with strength $c_{h}$.

\section{ENCODING OF THE PERCEPTUAL SEQUENCE}

\section{A. Repeated items}

The problem of how repeated items are encoded and retrieved has challenged theories of serial order. Ordinal theories, to which the dynamic field model belongs, apply a gradient of item activations in memory to represent order among items without recourse to any associative process (for review see [26]). Typical connectionist implementations assume that individual items are represented by just a single node [36], [37]. However, the repetition of an item in the input stream will increase its activation relative to neighboring items, potentially disrupting the order representation. To overcome this problem, additional processing mechanisms providing positional information have been integrated such as an association of items with a smoothly varying control signal [36] or socalled "rank-order neurons" that specifically respond to the first, second or third presentation of a particular item [38] (for a DNF implementation of a position code see [39]). In the DNF model, there is no need to distinguish the processing of repeated and non-repeated cues due to the feedback inhibition from the "on memory" field to the perceptual field. Its primary role is to destabilize an existing bump such that a new color event can be processed. At the same time, the additional inhibition from the memorized color guarantees that the new bump evolves at a different position even if the color is repeated. The only assumption we have to make is that only a small portion of all neurons tuned to the specific hue value evolves suprathreshold activity pattern in response to a single input presentation. In a two-dimensional field in which the $x$-coordinate represents color and the $y$-coordinate represents item repetitions, a localized input representing a specific hue value takes the shape of a ridge [40], [41]. To integrate the computational mechanism for repeated items in a onedimensional field, we assume that the camera input activates a part of the field which is much larger than the width of a single bump. Since the exact shape of the transient input does not matter, we use a rectangular input for simplicity. The field thus appears to be segmented in blocks, each representing a specific hue value. Importantly, this block structure does not affect the buildup of the activation gradient in $u_{M_{o n}}$. There is no difference whether the currently processed hue value is repeated or not since only the time matters when the localized activity reaches the threshold. Fig. 2 shows a snapshot of sequence encoding in which a R-event and a G-event are already memorized as shown by the two bumps in $u_{M_{o n}}$, and a second $\mathrm{R}$ cue is currently processed in $u_{P_{o n}}$. As can be clearly seen, the position of the bump representing the second $\mathrm{R}$ event appears to be shifted to the left of the neurons getting feedback inhibition from the memory of the first $\mathrm{R}$ event.

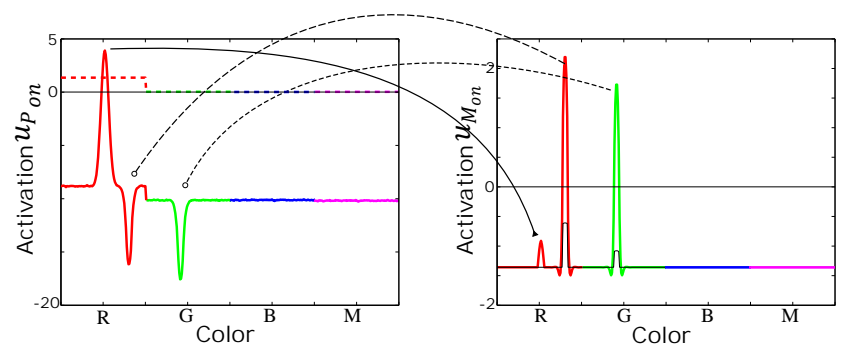

Fig. 2. Left: snapshot of activation (solid line) in the perceptual field, $u_{P_{o n}}$, in the presence of external input colored dashed line) indicating an R-event. Right: snapshot of activation in the "on" sequence memory field, $u_{M_{o n}}$, black dotted lines indicate inhibitory and black solid lines excitatory connections between the two fields.

\section{B. Role of preshaping}

The memory in $u_{M_{o n}}$ is the result of the exposure to repeated demonstrations of the entire sequence (purely perceptual learning [42]). The increasing perceptual experience with the task is reflected by an increasing baseline activity of neural populations [43] that have processed item information in preceding demonstrations. In the model, this preshaping mechanism is the result of a fading memory trace in $u_{M T}$ which receives the activation pattern in $u_{M_{o n}}$ as excitatory input. We assume that without the intention to actively recall the sequence, the activity pattern in $u_{M_{o n}}$ is reset to resting state by the stop signal at the end of every sequence demonstration. The time constant of the field dynamics in $u_{M T}$, on the other hand, is chosen to ensure that the memory trace may accumulate evidence from previous demonstrations. The functional role of this preshaping mechanism is to boost the processing of "expected" items. Since only one bump may exist in $u_{P_{o n}}$ at a time, an input which is processed when 
there exists still suprathreshold activity from the preceding one, or an input which is too weak, may fail to drive a suprathreshold activity pattern in $u_{P_{o n}}$. Consequently, the item is not represented in $u_{M_{o n}}$. The probability of such an encoding error is particularly high for repeated items due to the inhibition from neighboring neurons or the feedback inhibition from the corresponding population in $u_{M_{o n}}$ (for experimental evidence of so-called "repetition blindness" see [44]).

Fig. 3 presents results of a model simulation in which a sequence of five color events including one repetition of $\mathrm{R}$ is demonstrated in three consecutive trials. In the left panels, the pre-activated populations of the perceptual field at the beginning of the second and third trial reflect the growing perceptual experience with the task. In the right panels, the temporal evolution of supratreshold population activity in $u_{M_{o n}}$ is compared relative to the onset of the individual color event (vertical line). The evolution is characterized by two distinct phases. In response to the transient input from $u_{P_{o n}}$, the activity first reaches a peak and then decays. This is followed by a phase of monotonically increasing population activity due to the threshold accommodation dynamics. In the first demonstration, the second input, which is a repetition of the first, is not represented. At the moment when the second input is applied to the perceptual field, the population representation of the first stimulus is still suprathreshold (not shown), resulting in an inhibition of neighboring neurons. As a result, the second input fails to trigger a suprathreshold response at a position which receives this lateral inhibition. The situation is different in the second trial in which the processing of all inputs except the second already starts from an activation level closer to threshold. The temporal evolution of the transient population response representing the first color event is now fast enough so that the second repeated input may trigger a bump. The activation gradient established in $u_{M_{o n}}$ at the end of this trial reflects the correct serial order of all five items. However, the variability in the time delay between stimulus onset and the moment when the population response reaches threshold is relatively high, indicating that the relative timing of events is still not well represented in memory. As can be seen in the evolution of the population activities in the third trial, a larger pre-activation of populations in $u_{P_{o n}}$ due to a larger input from $u_{M T}$ significantly reduces this variability, resulting in a nearly identical delay for all item memories.

\section{SEQUENCE RECALL: DIFFERENT EXECUTION SPEEDS}

The information stored in the activation gradient can be recalled with the ramp-to-threshold dynamics which starts at $t_{\text {start }}=0$ from pre-activated population representations. As can be seen in Fig. 4(A), the preshape strength at $t_{\text {start }}$ corresponds to the serial position of each item in the sequence [24]. The release of inhibition for all subpopulations governed by equation (5) results in a parallel increase of activity until the threshold for triggering a bump is reached, starting with the subpopulation representing the first item and ending with the subpopulation representing the last item. To show that the parallel ramping dynamics also preserves the information about the relative timing of events, we compare in Fig. 4(C)

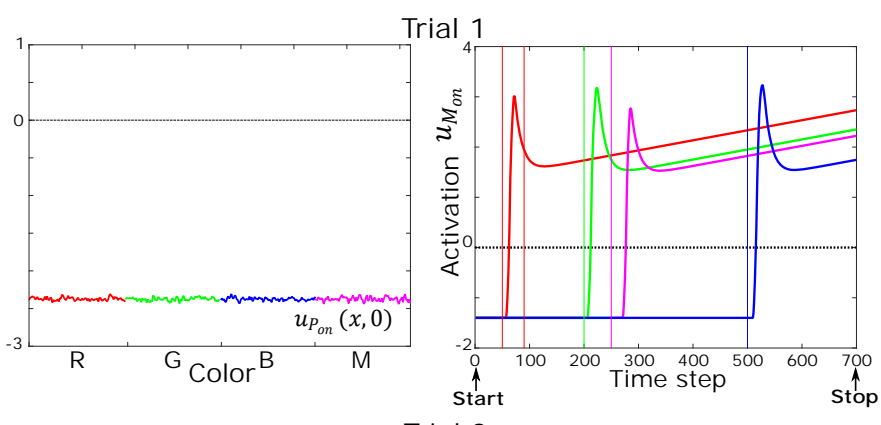

Trial 2

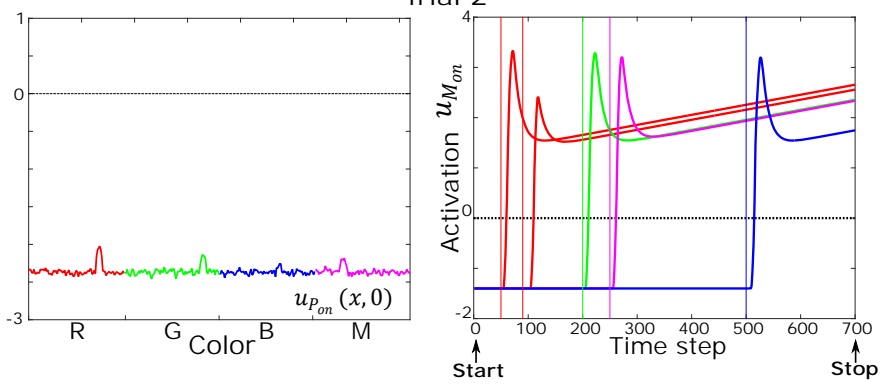

Trial 3
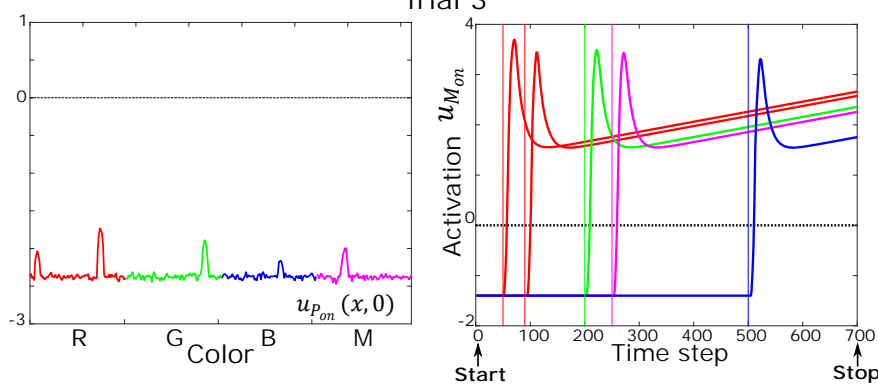

Fig. 3. Three successive sequence demonstrations starting at $t=0$ and ending at $t=700$. Left: snapshots of the initial state of the perceptual field, $u_{P_{o n}}$, in the three trials. Right: temporal evolution of the activity of each subpopulation representing the different sequence elements in the "on" sequence memory field, $u_{M_{o n}}$.

the bump-onset-asynchrony (BOA) between successive color events for the neural trajectories in $u_{M_{o n}}$ (Fig. 3, Trial 3) and in Fig. 4(A). The amount of time between successive zero crossings expressed in percentage of sequence duration is nearly identical for the encoding and the recall dynamics. It is important to note that choosing a higher readout threshold would not change the relative timing pattern. It would, however, increase the processing delay relative to the demonstrated event timing (with onset at $t=0$ ) as indicated by the vertical lines in Fig. 4(A). Different recall speeds can be achieved by adjusting the slope $\beta_{D}$ of the ramping dynamics (5). Fig. 4(B) shows a faster recall in which the activity profile of all subpopulations appears to be shifted to the left since the threshold for triggering a bump is reached earlier. A comparison of the bars in panel $(\mathrm{C})$ reveals that the pattern of relative event timing (BOA) encoded in the activation gradient is still preserved.

It is interesting to note that the purely perceptual acquisition of the sequence knowledge may be directly used to reproduce the sequential regularities in overt motor behavior. The prerequisite is that an associative coupling between a specific color event and the corresponding motor response has been 
already established and motor delays are negligible (for the example of a robot learning to execute a color-coded melody on a keyboard see [13]).
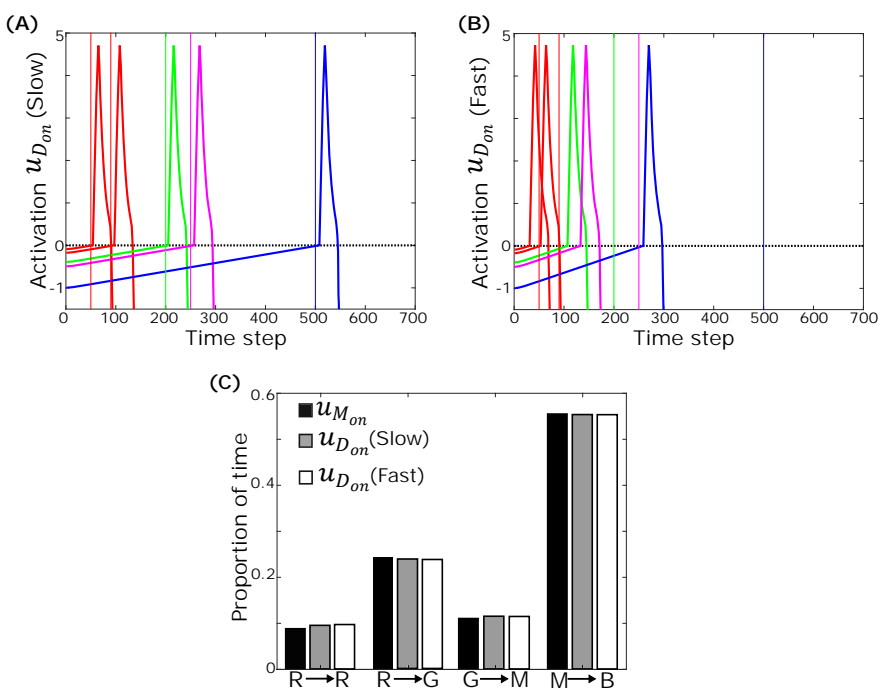

Fig. 4. Temporal evolution of activity representing each color event in $u_{D_{o n}}$ for different recall speeds: (A) $\beta_{D}=0.001$ and (B) $\beta_{D}=0.002$. Comparison of BOAs of successive events during encoding, $u_{M_{o n}}$, and during recall, $u_{D_{o n}}$, for the slow and the fast $(\mathbf{C})$ condition. Note that the relative timing pattern is preserved.

\section{MODEL EXTENSIONS}

\section{A. Synchronization with external events}

The results in Fig. 3 show that the active temporal anticipation of events based on prior experience [45] lags behind the demonstrated pattern of event timing. While this is not problematic for tasks in which the planning of future events is associated with a self-timed sequence onset, other tasks such as a series of fluent object handovers to an operator require the capacity to synchronize the motor timing with external reference cues signaling the expected timing pattern. Since for a given ramping dynamics, the initial baseline state controls the time it takes to reach threshold, we adapt the baseline activity based on a comparison between the neural trajectories of a recalled item (prediction) and a reference signal (perception). To explain the neural computations, we use as a simple example the neural representations of the first sequence element centered at position $x_{1}$ in $u_{D_{o n}}$ and an external color cue centered at position $x_{E}$ in $u_{P_{o n}}$. The initial value $h_{D_{0}}$ of the ramping activity is adapted using the following dynamics:

$$
\begin{array}{r}
\frac{\mathrm{d} h_{D_{0}}}{\mathrm{~d} t}(t)=\beta_{D_{0}}\left[1-f\left(u_{D_{o n}}\left(x_{1}, t\right)\right) f\left(u_{P_{o n}}\left(x_{E}, t\right)\right)\right] \\
\times\left[f\left(u_{D_{o n}}\left(x_{1}, t\right)\right)-f\left(u_{P_{o n}}\left(x_{E}, t\right)\right)\right]
\end{array}
$$

where $\beta_{D_{0}}>0$ is the adaptation rate. The adaptation takes place during the time window when the activity of the first population reaches the threshold and the neural trajectory of the other population is still subthreshold. Fig. 5 shows the results of two model simulations in which the external reference signal is presented at $t=100$ and the prediction of the first event appears to be too early (panel A) or delayed
(C)

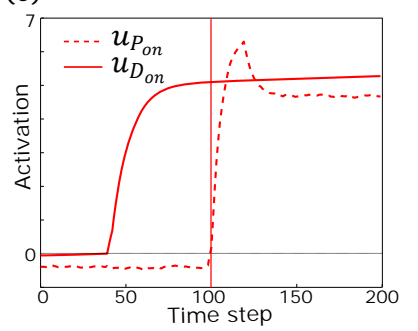

(A)

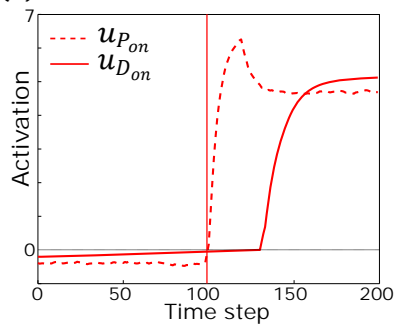

(B)

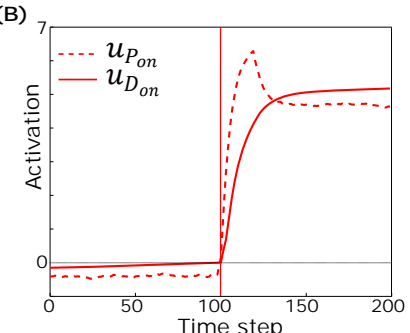

Fig. 5. Resting state adaptation in $u_{D_{o n}}$. Examples of a $\mathrm{R}$ event which is recalled either too early $(\mathbf{A})$ or too late $(\mathbf{B})$ relative to the processing of the reference input (dashed line). (C) After adaptation of the baseline activity, $h_{D_{0}}$, in the decision field, the recall occurs in both cases in synchrony with the respective reference signal.

(panel B) relative to reference time. The resting state dynamics (6) applied in a single trial guarantees in both cases that in the next sequence recall the bump attractors evolve in synchrony, as shown in panel $\mathrm{C}$. Since the resting state is adapted for the whole field, the neural trajectories of all sequence elements appear to be shifted by the same amount of time. The global adaptation thus represents an efficient means to compensate in a single trial similar temporal offsets of all memorized items (Fig. 3). It is also possible to apply equation (6) locally to adjust the height of individual memory bumps of the gradient in $u_{M_{o n}}$. In the object transfer task, for instance, motor delays in the object grasping that may differ from object to object have to be compensated to guarantee a fluent interaction. Fig. 6 shows an example of the formation of a "motor memory" in which the memory strength of individual items still have to be increased (G-event) or decreased (M-event) based on a comparison with the temporal processing of the respective reference signal. Fig. 6(A) compares the preshaping at time $t=0$ in $u_{D_{o n}}$ due to the input from $u_{M_{o n}}$ before (solid line) and after (dashed line) the local h-adaptation in $u_{M_{o n}}$. A comparison of the temporal evolution of recall for both cases, shown in panels (B) and (C), reveals that the relative higher pre-activation of the $\mathrm{G}$-event and the relative lower preactivation of the M-event compensate the temporal mismatch observed in the first sequence recall.

\section{B. Encoding of event duration}

Fig. 7 sketches an extended version of the model described in Section II in which also the duration of color events becomes memorized. Fluent object handovers minimize the waiting time at the exchange position for the "giver" and the "receiver". It is thus important that a robotics assistant is able to measure the expected exchange duration. The basic idea is that during demonstration the robot camera not only registers 

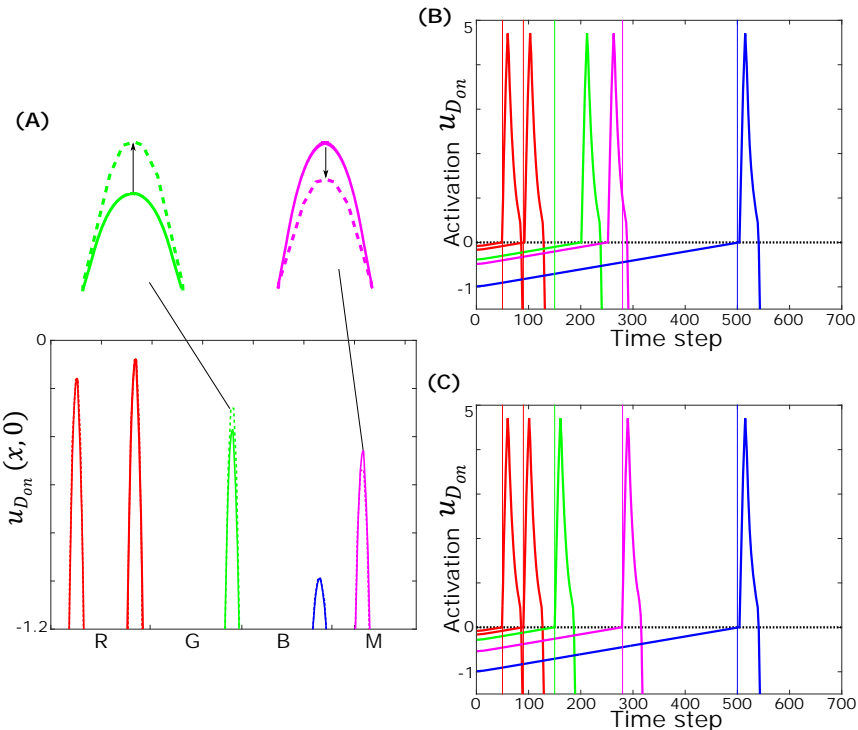

Fig. 6. Local h-adaptation of individual event memories to compensate initial timing errors. (A) The pre-activation in the decision field, $u_{D_{o n}}(x, 0)$, is compared before (solid line) and after (dashed line) the memory adaptation of the G-event and the M-event, respectively. To better visualize the change in preshape strength, the two relevant pre-activations are magnified. (B) Time course of sequence recall before adaptation. The M-event is recalled too early and the G-event too late. (C) After adaptation, all items are recalled at the expected event times.

the onset of a specific color input at the exchange position (object reaches position) but also the offset of the visual signal (object leaves position). It is then straightforward to represent in the DNF model also the duration of a color event by assuming that like for the "on" signals also the sequence of "off" signals is represented by an activation gradient in an "off memory" field, $u_{M_{o f f}}$. The autonomous sequence recall then starts at $t_{\text {start }}=0$ from a pre-activated state in the associated "off decision" field, $u_{D_{o f f}}$. Each evolving bump in $u_{D_{o f f}}$ becomes destabilized through the excitatory/inhibitory connections to corresponding populations in the working memory field $u_{W M}$. The only modification for the recall of the "on" sequence is that there are no excitatory connections from $u_{D_{o n}}$ to $u_{W M}$ (compare Fig. 1). A bump in $u_{W M}$ associated with a specific "off" signal also destabilizes through inhibitory connections the corresponding "on" signal representation in $u_{D_{o n}}$. Fig. 8 depicts the time course of the independent serial recall in the two decision fields, $u_{D_{o n}}$ (top panels) and $u_{D_{o f f}}$ (bottom panels), for two different scenarios. The baselines in both fields have been chosen, using the adaptation dynamics (6), to guarantee a recall in synchrony with the demonstrated timing patterns. The ramping dynamics starts in both decision fields with the presentation of the start signal at $t_{\text {start }}=0$. The time courses of suprathreshold activities in $u_{D_{o n}}$ reflect event duration for a sequence with temporally non-overlapping color signals (Fig. 8(A)), and for a sequence in which the third event starts earlier but lasts longer than the forth (Fig. 8(C)). The latter case corresponds to an exchange of the rank order between the two items in $u_{M_{o f f}}$ relative to $u_{M_{o n}}$.

It is worth noting that an alternative way to encode event duration is to trigger the ramp-to-threshold dynamics in $u_{D_{o f f}}$

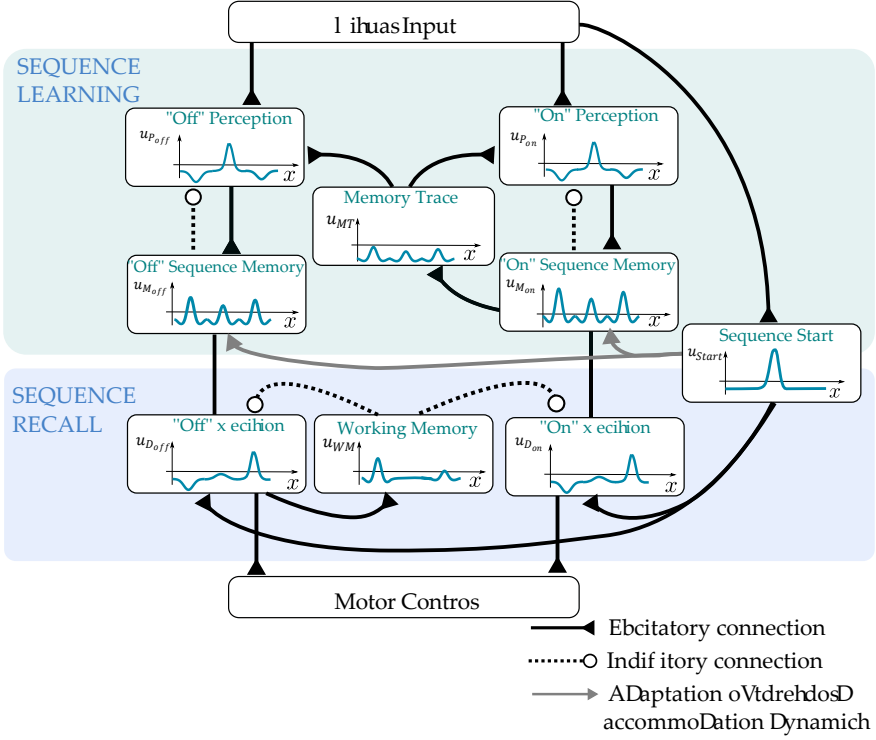

Fig. 7. Sketch of the extended DNF model able to learn and represent event duration defined by the time interval between the onset and the offset of a visual event. Note that the encoding and recall of the offset of a series of visual events follows the same neuro-computational principles used for the encoding and recall of the onset sequence. Dotted lines represent inhibitory connections, solid lines excitatory connections. The arrows from the sequence start field $u_{S t a r t}$ to the memory fields $u_{M_{o n}}$ and $u_{M_{o f f}}$ indicate that the time window for the threshold accommodation dynamics in $u_{M_{o n}}$ and $u_{M_{o f f}}$ is controlled by the presence of a bump in $u_{\text {Start }}$. For details see the text.

not simultaneously for all items at time $t_{\text {start }}$ but sequentially at the time when the population representation of the corresponding "on" signal reaches threshold. A prerequisite is that the preshape-threshold distance in $u_{D_{\text {off }}}$ has been adjusted to represent duration. This can be achieved by applying the local version of the adaptation dynamics (6) to the memory representations in $u_{M_{o f f}}[46]$.
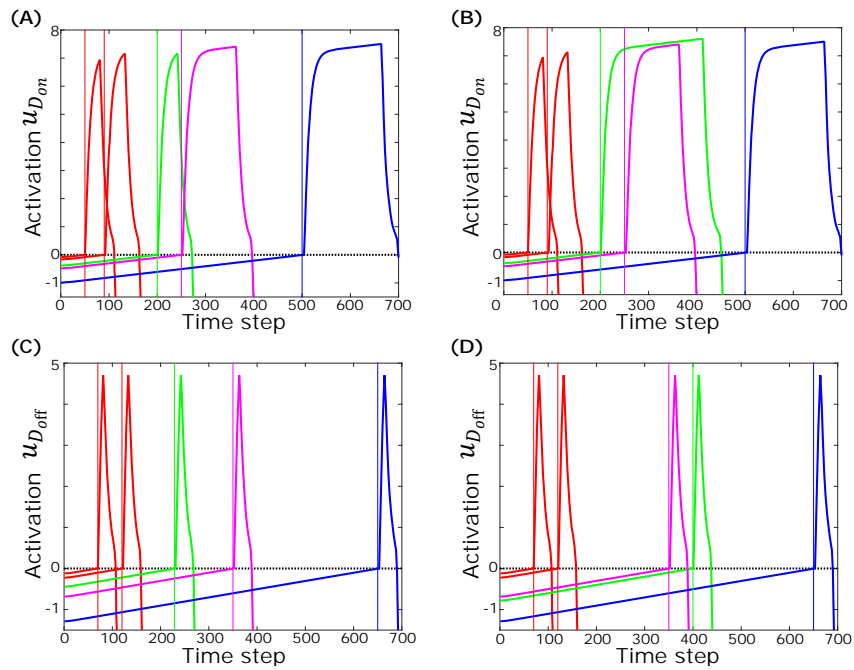

Fig. 8. (A) and (B) show the temporal evolution of activity representing each item the "on" decision field, and (C) and (D) how the temporal evolution of activity representing each item the "off" decision field, for the same sequence (R-R-G-M-B) with different event durations: 20-30-30-100-150 time steps and 20-30-200-100-150 time steps, respectively. $t_{o n}=0$ is the time of sequence onset. 


\section{SEQUENCE ERRORS AND PRECISION OF TIME MEASUREMENTS}

Traditionally, sequence recall errors and errors in estimating temporal intervals that are typically observed in experiments with humans and other animals have been used to distinguish different theories of sequence learning [47], [26] and interval timing [48], [49]. Introducing additive noise to the field equation (1) and the baseline dynamics (5), $c_{u}>0$ and $c_{h}>0$, allows us to address model robustness. Rather than trying to fit detailed error patterns that may depend on the exact nature and design of sequential tasks, we show in model simulations how specific serial order errors and time estimation distributions are explained by the dynamic field concepts.

\section{A. Transposition errors}

Erroneous sequence recall performance may have different origins. It may be the result of a mistake in stimulus encoding like in the example of Fig. 3, or may reflect a conflict on the sequence production level like for instance the occurrence of a repetition error when the response suppression due to the feedback inhibition from $u_{W M}$ is too weak. However, the prevalent error type found in serial recall tasks is a simple exchange with adjacent items in the planned sequence (transposition error, [47]). Like in other gradient based models of serial performance, random fluctuations in the decision field may corrupt the competition between neural representations with similar pre-activations. To choose the noise strengths, $c_{u}$ and $c_{h}$, we assume that after learning 1) transposition errors are relatively rare events, and 2) an error-free serial recall preserves the timing information encoded in the memory gradient. Fig. 9(A) shows a model simulation for an example sequence with five memorized events. The noise added to each field site causes random fluctuations in the ramp-tothreshold dynamics but the serial order is correctly reproduced as revealed by the transient population activation profiles in Fig. 9(B). Moreover, as shown in Fig. 9(C), the BOAs match well the relative timing pattern encoded in the memory gradient, with an error below 2.5\% for all four intervals. Fig. 10(A) depicts for the same example sequence and noise strengths a model simulation with transposition error. A premature recall of the fourth item is followed by the displaced third item. Interestingly, the model predicts a relation between the sequence production rate (controlled by the parameter $\beta_{D}$ of equation 5) and the percentage of such anticipatory errors in a given number of trials. Fig. 10(B) shows for a numerical experiment with 200 simulations of the stochastic field model an approximately linear increase of the error rate with speed $(r=0.938867, p<0.01)$.

\section{B. Variability in timing of consecutive events}

A hallmark of human timing of single, isolated intervals in the range of tens of milliseconds to several seconds is Weber's law [50]. It states that the variability in subjective duration estimation increases linearly with the mean of the distribution. Entire time estimation distributions thus superimpose when the time axis is normalized with respect to the mean. As a measure

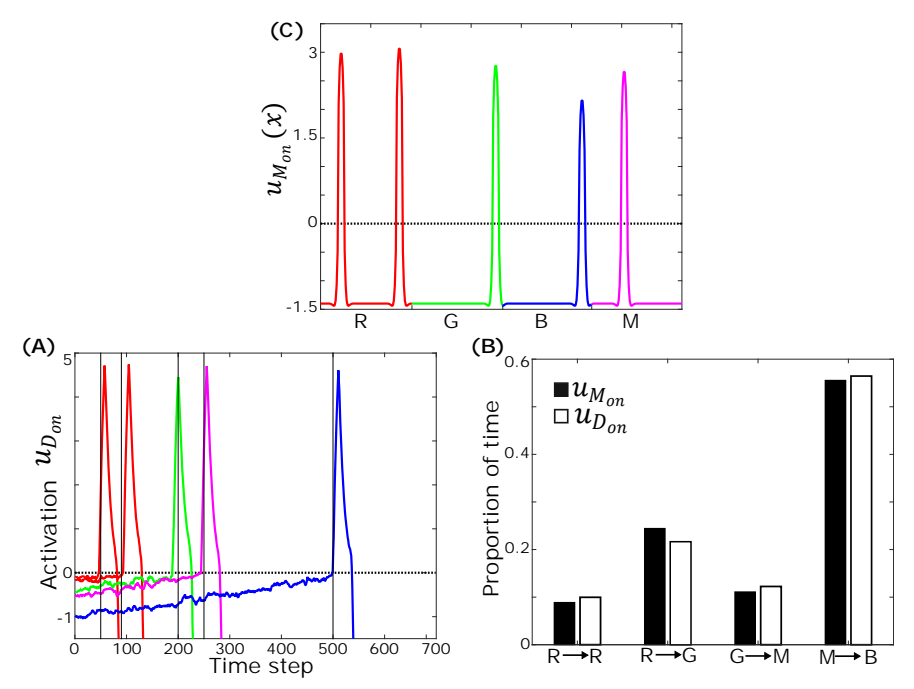

Fig. 9. (A) Stable activation pattern corresponding to the correct memory of a sequence (R-R-G-M-B). (B) The temporal evolution of population activity in $u_{D_{o n}}$ representing the different sequence elements is shown. The vertical lines indicate the time of cue presentation. (C) Relative timing of successive events in $u_{M_{o n}}$ and in $u_{D_{o n}}$. Parameters for the noise in $u_{D_{o n}}$ given by equation (8) are $c_{u}=0.04, w_{e x c}=1, \sigma_{\text {exc }}=0.8, w_{i n h}=0$, the noise strength for the h-dynamics (5) is $c_{h}=0.001$.
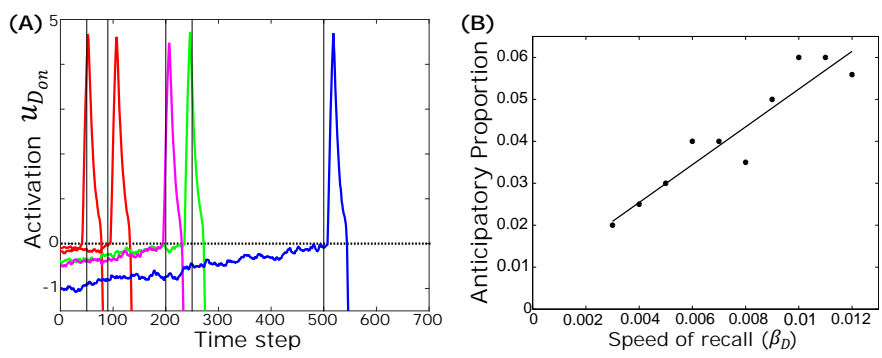

Fig. 10. (A) The temporal evolution of population activity in $u_{D_{o n}}$ representing an anticipatory error is shown (B) Correlation between the percentage of trials with anticipatory errors and the speed of serial recall in 200 model simulations. The noise parameter values are the same as in Fig. 9.

of relative precision, the coefficient of variation (or Weber fraction), $C V=\sigma / T$, is usually used where $\sigma$ is the standard deviation of the underlying distribution and $T$ is the interval being measured or produced. There are, however, also wellknown violations of Weber's law (for an overview see [51]). Important for the present context, a recent study [52], in which subjects learned to reproduce a sequence of finger taps with the correct order and timing, found a systematic decrease in $C V$ as timed intervals increase. Moreover, the findings supports the notion of a continuous timing of all consecutive events in relation to sequence onset at $t=0$, as opposed to a resetting of an interval timer at each event. In the decision field of the stochastic DNF model, the time when the population activity reaches threshold varies on a trial-by-trial basis due to the continuous noise. Fig. 11(A) shows for all consecutive events the distribution of their expected timing obtained in $N=1000$ recall simulations. The overlaid normalized Gaussian fits of the distributions do not superimpose, but reveal in line with the experimental findings a decrease in the standard deviation along the sequence (Fig. 11(B)). The plot of the Weber fractions in Fig. 11(C) confirms the violation of Weber's law. 

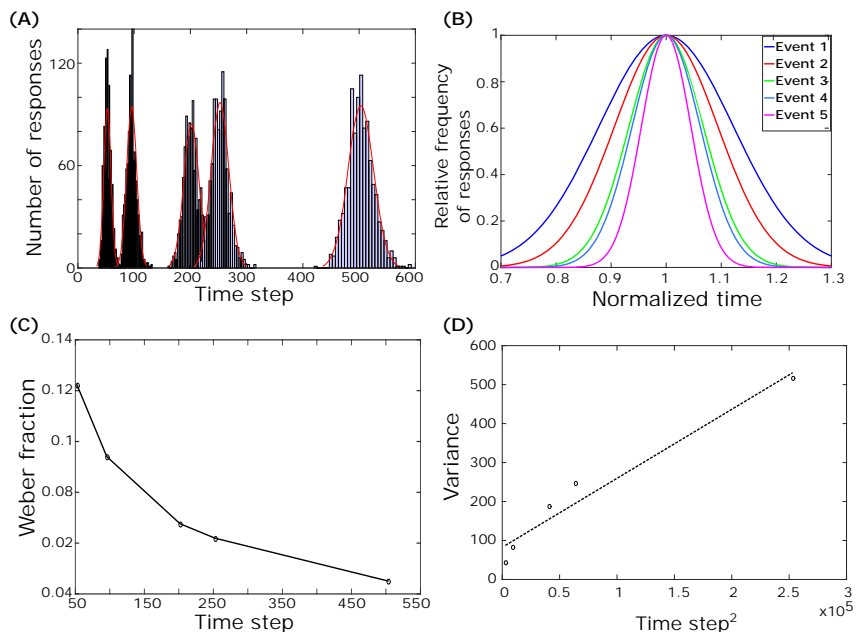

Fig. 11. (A) Distribution of response times for each event. (B) Overlaid normalized Gaussian fits from (A). Each distribution has the $x$-axis divided by its mean and their $y$ values divided by the peak value. (C) Weber fraction (=coefficient of variation) as a function of mean response time. (D) Variance as a function of mean response time squared. Noise parameters for the field dynamics (8) are $c_{u}=0.02, w_{\text {exc }}=1, \sigma_{\text {exc }}=0.8, w_{i n h}=0$ and the noise strength for the h-dynamics (5) is $c_{h}=0.02$.

As suggested in [52], a fit of a generalized version of the law, which states that the variance $\sigma^{2}$ increases with $T^{2}$, captures the model results well (Fig. 11(D)).

\section{DISCUSSION}

In this computational study, we tested the idea that persistent neural population activity that varies monotonically with elapsed time [21] may support the rapid learning of ordinal and interval properties of sequences. The simulations of the DNF model show that the relative level of persistent activity among subpopulations, established by the field dynamics in very few demonstrations of a fairly complex sequence, robustly encodes the order and relative timing of events. While the two task variables, cue identity and elapsed time, share a common neural substrate, they are controlled by different neural mechanisms [22], recurrent interactions and threshold accommodation, respectively. As shown in Fig. 3 and Fig. 5, the model may account for substantial changes in the temporal pattern while the ordinal structure remains constant. This is in line with sequence learning experiments, showing that the correct serial order is typically established before the temporal structure [5]. The recall of the stored information starts from a pre-activated state, reflecting the rank position of each event, with a consistent increase of population activity until a pre-defined threshold, associated with the predicted event timing, is reached. While the preshape pattern and the parallel activation of all subpopulations are consistent with "ordinal models" of serial order [26], the climbing neural activity is consistent with "ramping models" of interval timing (for review see [53]). Most ramping models rely on balanced feedback mechanisms on the network or the cellular level to generate temporal integrator-like activity and adjust it to varying temporal context [54], [55]. In the DNF model, the time course of decaying global inhibition in the decision field is controlled by the integration of an external input representing the memory of the start signal. Due to the input integration, the stochastic field model with additive noise, used to address the precision of temporal expectations (Fig. 11), can be discussed within a drift-diffusion decision theoretic framework [28].

To distinguish the DNF approach from related neuro-inspired approaches to sequence learning, it is useful to understand the implemented neural mechanisms from a dynamical systems perspective. Rapid learning and temporal flexibility are achieved without any change in the network structure through manipulations of inputs and initial conditions that shape the neural trajectory towards an existing bump attractor solution (for discussion see [29]). Approaches based on recurrent neural networks (RNN), on the other hand, assume that learning manifests in synaptic plasticity using Hebbian learning rules (e.g., [56], [3], for an overview of modern RNN-based sequence learning models see [57]). For instance, in the study addressing the learning of a precisely timed finger tapping sequence [52], the authors used a "population clock" model to explain their experimental findings. To read out event times, output units are trained to recognize a specific pattern of the distributed time-varying activity in the network. Synaptic plasticity within the RNNs is also required to guarantee a robust and reproducible initiation of the neural trajectory by external inputs [58]. We leave a more detailed comparison with state-of-the-art sequence learning models based on synaptic plasticity for future work.

The emphasis on activation based learning does not exclude however that in real-world applications the DNF-model may also involve structural changes. In a recent robotics experiment [59], we have implemented the idea that the two learning mechanisms may have complementary roles. We used fast activation based learning to store in separate neural fields the order of a sequential task demonstrated by users with different order preferences. The spontaneous recall of the gradientbased memories is then taken as input to a recurrent neural network to gradually establish through Hebbian learning longterm associations between subtasks. The ultimate goal was to smoothly integrate into the network structure generalized task knowledge from different user demonstrations. The storage of different order-timing information in separate fields can be also directly used to endow the robot with the capacity to flexibly adapt to different human co-workers in a shared task. All one has to assume is that the excitatory connections between the various demonstration-specific memory fields and the recall field are gated by an additional (visual) cue which identifies a specific co-worker.

For robotics implementation of DNF models it is important to stress that the processing of sensory information in recurrent networks, as implemented in the perceptual field, $u_{P_{o n}}$, is known to greatly increase the signal-to-noise ratio [32]. This contributes to a robust encoding and recall of sequential information by the intrinsic network mechanisms even for relatively weak and noisy inputs.

The results of the stochastic model simulations in section VI-A confirmed a well known limitation of ordinal models that exploit relative activation levels to represent serial order. Noise 
in the system limits the storage capacity. With the present parameter setting, we have successfully tested up to 10 color events that can be reliably recalled from a single gradient established by the threshold dynamics. Since there is no difference in the processing of repeated and non-repeated items, the order pattern can be quite complex (e.g., GGRRRRMGGM). While a rare occurrence of noise-induced transposition errors (Fig.10) might be acceptable for a learning robot, it is clear that introducing more elements in a sequence of fixed duration will increase the error probability. We are currently exploring for our robotics work how "chunking" mechanisms [9] for sequences with more items can be integrated into the DNF model of rapid sequence learning to cope with this challenge [59].

A further shortcoming of the present model is that the predefined growth rates of the threshold accommodation (4) and the ramping activity (5) are not automatically adapted to behaviorally relevant time scales that may range from a few seconds to minutes. One way to achieve such a temporal scaling is to assume the existence of a neural population that "measures" (e.g., during the first sequence demonstration) with a fixed buildup rate of the threshold accommodation the duration of the whole sequence defined by the start and stop signals. The amplitude of the "measuring" bump then sets the buildup rate with an inverse relationship, that is, the higher the bump the shallower are the slope of the ramp [29].

To guide sequence learning in real-world robotics applications it is highly desirable to build object representations over a larger number of feature dimensions that the camera is able to detect. In [40], an architecture of coupled dynamic fields representing simple features, such as color, size and aspect ratio is presented that provides an efficient object recognition. It could be integrated into the sequence learning model when no distinctive individual feature dimension exists.

\section{CONCLUSION}

The motivation to search for neuro-inspired solutions to the problem of learning a sequence with time constraints is the efficient and highly flexible capacity of humans to coordinate the ordering and timing of activities with co-workers in a shared task. To guarantee a satisfying and pleasant user experience, an autonomous robot engaged in cooperative work with a human should be able not only to process, plan and adapt the order but also onset and duration of sensorimotor events. Since the important role of time in cognition is to a large extent neglected in current robotics research [16], the smooth integration of the temporal dimension in the neurodynamics approach holds the potential to guide the development of a new generation of cognitive robots. We are currently testing the learning model as part of a DNF based control architecture for natural human-robot interaction [17], [18] in a series of object handovers. The robot has to adapt the learned ordertiming representations to changing preferences of the coworker or additional task constraints while preserving a tight synchronization of activities.

\section{APPENDIX A \\ MODEL EQUATIONS}

Since the model equations for the processing of the on and the off signals are identical, we refer in the following only to the on field equations. The dynamics of the perceptual field $u_{P_{o n}}$, the sequence memory field $u_{M_{o n}}$ and the memory trace field $u_{M T}$ are governed by the following equations, respectively:

$$
\begin{array}{r}
\tau_{P_{o n}} \frac{\partial u_{P_{o n}}(x, t)}{\partial t}=-u_{P_{o n}}(x, t)+u_{M T}(x, t) \\
+S_{o n}(x, t)+\int w_{l a t}(|x-y|) f\left(u_{P_{o n}}(y, t)\right) \mathrm{d} y \\
-\int w_{o s c}(|x-y|) f\left(u_{M_{o n}}(y, t)\right) \mathrm{d} y+c_{u} \xi(x, t) .
\end{array}
$$

The noise $\xi(x, t)$ is given by:

$$
\xi(x, t)=\int w_{\text {lat }}(|x-y|) \mathrm{d} \delta(x, t) \mathrm{d} y
$$

where $\mathrm{d} \delta(x, t)$ is a spatially uncorrelated Wiener process filtered with the Gaussian kernel $w_{\text {lat }}$.

$$
\begin{array}{r}
\tau_{M_{o n}} \frac{\partial u_{M_{o n}}(x, t)}{\partial t}=- \\
\quad u_{M_{o n}}(x, t)+h_{M_{o n}}(x, t) \\
+u_{P_{o n}}(x, t) f\left(u_{P_{o n}}(x, t)\right) \\
+\int w_{o s c}(|x-y|) f\left(u_{M_{o n}}(y, t)\right) \mathrm{d} y, \\
\tau_{M T} \frac{\partial u_{M T}(x, t)}{\partial t}=-u_{M T}(x, t)+h_{M T} \\
+\lambda_{M T} u_{M_{o n}}(x, t) f\left(u_{M_{o n}}(x, t)\right) .
\end{array}
$$

The perceptual field $u_{P_{o n}}$ receives a localized visual input $S_{\text {on }}(x, t)$ which we chose for simplicity as being of rectangular shape with constant amplitude. The width of this input is assumed to be much larger than the width of an individual bump. The connection functions $w_{\text {lat }}$ and $w_{\text {osc }}$ determine the coupling between neurons within the field. For the perceptual field and the "on" decision field in which only one bump at a time should evolve, we use a kernel of lateral inhibition type given by (2). To enable multi-bump solutions in the memory fields, we use a kernel with oscillatory rather than monotonic decay given by (3).

The constant $h_{M T}<0$ is the global resting level for the past memory field $u_{M T}$. The parameter $\lambda_{M T}$ represents the rate at which the subthreshold pattern of the past sequence memory is built. To ensure that the building is stronger than the forgetting, in successive sequence presentations, the rate $\lambda_{M T}$ must be larger than 1 . The threshold accommodation of $u_{M_{o n}}$ is governed by equation (4).

The dynamics of the decision field $u_{D_{o n}}$, the working memory field $u_{W M}$, and the sequence start field $u_{\text {Start }}$ are governed by the following equations, respectively:

$$
\begin{array}{r}
\tau_{D_{o n}} \frac{\partial u_{D_{o n}}(x, t)}{\partial t}=-u_{D_{o n}}(x, t)+h_{D}(t) \\
+\int w_{o s c}(|x-y|) f\left(u_{D_{o n}}(y, t)\right) \mathrm{d} y \\
-\int w_{o s c}(|x-y|) f\left(u_{W M}(y, t)\right) \mathrm{d} y+u_{M_{o n}}(x),
\end{array}
$$




$$
\begin{array}{r}
\tau_{W M} \frac{\partial u_{W M}(x, t)}{\partial t}=-u_{W M}(x, t)+h_{W M} \\
+u_{D_{\text {state }}}(x, t) f\left(u_{D_{\text {state }}}(y, t)\right) \\
+\int w_{\text {osc }}(|x-y|) f\left(u_{W M}(y, t)\right) \mathrm{d} y .
\end{array}
$$

In simulations of equation (12), $u_{D_{\text {state }}}=u_{D_{o n}}$ when only the "on" sequence is recalled and $u_{D_{\text {state }}}=u_{D_{\text {off }}}$ when the "off" and "on" sequence are both recalled.

The baseline activity $h_{D}(t)$ evolves continuously in time following (5), the baseline $h_{W M}<0$ is a constant.

\section{APPENDIX B}

\section{NUMERICAL METHOD AND SIMULATION PARAMETERS}

Numerical simulations were done in MATLAB using a forward Euler method with parameters given in Table I. We assume a finite domain with length $L=360$ that is discretized into $n=7200$ equal intervals of size $\mathrm{d} x$ so that $\mathrm{d} x=\frac{L}{n}=0.05$. To find $u(x, T)$ where $T>0$, we discretize time $T$ into $m$ equal steps of size $\Delta_{T}=1$. To compute the spatial convolution of $w$ and $f$ we employ the MATLAB function conv.

\begin{tabular}{|c|c|}
\hline \multicolumn{2}{|r|}{ "On/Off" Perception, $u_{P_{o n}}$} \\
\hline$\tau_{P_{o n}}$ & 6 \\
\hline$w_{\text {lat }}(x)$ & $w_{\text {exc }}=4, \sigma_{\text {exc }}=3.4, w_{i n h}=2$ \\
\hline$w_{o s c}(x)$ & $A=2, b=0.25, \alpha=0.052$ \\
\hline$\epsilon \xi(x, t)$ & $\epsilon=0.025, w_{e x c}=1, \sigma_{e x c}=0.5, w_{i n h}=0$ \\
\hline \multicolumn{2}{|r|}{ "On/Off" Sequence Memory, $u_{M_{o n}}$} \\
\hline$\tau_{M_{o n}}$ & 14 \\
\hline$h_{M_{o n}}(x, t)$ & $\beta_{M}=0.001, \int_{\Omega} f\left(u_{\text {Start }}(x)\right) \mathrm{d} x=2, h_{M_{0}}=-1.4$ \\
\hline$w_{o s c}(\mathrm{x})$ & $A=1, b=0.72, \alpha=0.52$ \\
\hline \multicolumn{2}{|r|}{ Memory Trace, $u_{M T}$} \\
\hline$\tau_{M T}$ & 6000 \\
\hline$h_{M T}$ & -1.4 \\
\hline$\lambda_{M T}$ & 1.5 \\
\hline \multicolumn{2}{|r|}{ "On/Off" Decision, $u_{D_{o n}}$} \\
\hline$\tau_{D_{o n}}$ & 10 \\
\hline$h_{D}(t)$ & $\beta_{D}=0.001, \int_{\Omega} f\left(u_{\text {Start }}(x)\right) \mathrm{d} x=2$ \\
\hline$w_{\text {osc }}(x)$ & $A=3.18, b=0.9, \alpha=0.9$ \\
\hline \multicolumn{2}{|r|}{ Working memory, $u_{W M}$} \\
\hline$\tau_{W M}$ & 12 \\
\hline$h_{W M}$ & -1.8 \\
\hline$w_{o s c}(x)$ & $A=3.18, b=0.9, \alpha=0.9$ \\
\hline
\end{tabular}

TABLE I

PARAMETER VALUES OF THE FIELD EQUATIONS.

\section{ACKNOWLEDGMENT}

The work received financial support from FCT (Portuguese Foundation for Science and Technology) through the $\mathrm{PhD}$ fellowship PD/BD/128183/2016, European Structural and Investment Funds in the FEDER component, through the Operational Competitiveness and Internationalization Programme (COMPETE 2020) and national funds, through the FCT projects PTDC/MAT-APL/31393/2017 (NEUROFIELD) and POCI-01-0247-FEDER-039334, and RD Units Project Scope: UIDB/00319/2020 and UIDB/00013/2020.

\section{REFERENCES}

[1] K. S. Lashley, The problem of serial order in behavior. Bobbs-Merrill Oxford, United Kingdom, 1951, vol. 21.

[2] A. Bubic, D. Y. Von Cramon, and R. I. Schubotz, "Prediction, cognition and the brain," Frontiers in human neuroscience, vol. 4, p. 25, 2010.

[3] P. F. Dominey, "A shared system for learning serial and temporal structure of sensori-motor sequences? evidence from simulation and human experiments," Cognitive Brain Research, vol. 6, no. 3, pp. $163-$ 172, 1998.

[4] R. I. Schubotz and D. Y. von Cramon, "Interval and ordinal properties of sequences are associated with distinct premotor areas," Cerebral Cortex, vol. 11, no. 3, pp. 210-222, 2001.

[5] F. Ullén and S. L. Bengtsson, "Independent processing of the temporal and ordinal structure of movement sequences," Journal of neurophysiology, vol. 90, no. 6, pp. 3725-3735, 2003.

[6] J. C. Shin and R. B. Ivry, "Concurrent learning of temporal and spatial sequences." Journal of Experimental Psychology: Learning, Memory, and Cognition, vol. 28, no. 3, p. 445, 2002.

[7] J. X. O'Reilly, K. J. McCarthy, M. Capizzi, and A. C. Nobre, "Acquisition of the temporal and ordinal structure of movement sequences in incidental learning," Journal of neurophysiology, vol. 99, no. 5, pp. 2731-2735, 2008.

[8] M. J. Nissen and P. Bullemer, "Attentional requirements of learning: Evidence from performance measures," Cognitive psychology, vol. 19 , no. 1 , pp. 1-32, 1987.

[9] P. Janata and S. T. Grafton, "Swinging in the brain: shared neural substrates for behaviors related to sequencing and music," Nature neuroscience, vol. 6, no. 7, pp. 682-687, 2003.

[10] C. Palmer and P. Q. Pfordresher, "Incremental planning in sequence production." Psychological Review, vol. 110, no. 4, p. 683, 2003.

[11] G. Schöner and J. Spencer, Dynamic thinking: A primer on dynamic field theory. Oxford University Press, 2016.

[12] W. Erlhagen and G. Schöner, "Dynamic field theory of movement preparation." Psychological review, vol. 109, no. 3, p. 545, 2002.

[13] F. Ferreira, W. Erlhagen, E. Sousa, L. Louro, and E. Bicho, "Learning a musical sequence by observation: A robotics implementation of a dynamic neural field model," in Development and Learning and Epigenetic Robotics (ICDL-Epirob), 2014 Joint IEEE International Conferences on. IEEE, 2014, pp. 157-162.

[14] E. Sousa, W. Erlhagen, F. Ferreira, and E. Bicho, "Off-line simulation inspires insight: a neurodynamics approach to efficient robot task learning," Neural Networks, vol. 72, pp. 123-139, 2015.

[15] W. Wojtak, F. Ferreira, L. Louro, E. Bicho, and W. Erlhagen, "Towards temporal cognition for robots: A neurodynamics approach," in 2017 Joint IEEE International Conference on Development and Learning and Epigenetic Robotics (ICDL-EpiRob). IEEE, 2017, pp. 407-412.

[16] M. Maniadakis and P. Trahanias, "Temporal cognition: a key ingredient of intelligent systems," Frontiers in neurorobotics, vol. 5, p. 2, 2011.

[17] E. Bicho, L. Louro, and W. Erlhagen, "Integrating verbal and nonverbal communication in a dynamic neural field architecture for human-robot interaction," Frontiers in neurorobotics, vol. 4, 2010.

[18] W. Erlhagen and E. Bicho, "A dynamic neural field approach to natural and efficient human-robot collaboration," in Neural fields. Springer, 2014, pp. 341-365.

[19] S.-i. Amari, "Dynamics of pattern formation in lateral-inhibition type neural fields," Biological cybernetics, vol. 27, no. 2, pp. 77-87, 1977.

[20] W. Erlhagen, A. Bastian, D. Jancke, A. Riehle, and G. Schöner, "The distribution of neuronal population activation (dpa) as a tool to study interaction and integration in cortical representations," Journal of neuroscience methods, vol. 94, no. 1, pp. 53-66, 1999.

[21] C. D. Brody, A. Hernández, A. Zainos, and R. Romo, "Timing and neural encoding of somatosensory parametric working memory in macaque prefrontal cortex," Cerebral cortex, vol. 13, no. 11, pp. 1196-1207, 2003.

[22] C. K. Machens, R. Romo, and C. D. Brody, "Functional, but not anatomical, separation of "what" and "when" in prefrontal cortex," The Journal of Neuroscience, vol. 30, no. 1, pp. 350-360, 2010.

[23] K. K. Sreenivasan, C. E. Curtis, and M. D'Esposito, "Revisiting the role of persistent neural activity during working memory," Trends in cognitive sciences, vol. 18, no. 2, pp. 82-89, 2014.

[24] B. B. Averbeck, M. V. Chafee, D. A. Crowe, and A. P. Georgopoulos, "Parallel processing of serial movements in prefrontal cortex," Proceedings of the National Academy of Sciences, vol. 99, no. 20, pp. 13172 13177,2002

[25] Y. Agam, D. Bullock, and R. Sekuler, "Imitating unfamiliar sequences of connected linear motions," Journal of Neurophysiology, vol. 94, no. 4, pp. 2832-2843, 2005. 
[26] B. J. Rhodes, D. Bullock, W. B. Verwey, B. B. Averbeck, and M. P. Page, "Learning and production of movement sequences: Behavioral, neurophysiological, and modeling perspectives," Human movement science, vol. 23 , no. 5 , pp. 699-746, 2004.

[27] R. Ratcliff and J. N. Rouder, "Modeling response times for two-choice decisions," Psychological science, vol. 9, no. 5, pp. 347-356, 1998.

[28] F. Balc1 and P. Simen, "A decision model of timing," Current opinion in behavioral sciences, vol. 8, pp. 94-101, 2016.

[29] E. D. Remington, S. W. Egger, D. Narain, J. Wang, and M. Jazayeri, "A dynamical systems perspective on flexible motor timing," Trends in cognitive sciences, vol. 22, no. 10, pp. 938-952, 2018.

[30] C. D. Brody, R. Romo, and A. Kepecs, "Basic mechanisms for graded persistent activity: discrete attractors, continuous attractors, and dynamic representations," Current opinion in neurobiology, vol. 13, no. 2, pp. 204-211, 2003.

[31] E. Bicho, W. Erlhagen, L. Louro, and E. C. e Silva, "Neuro-cognitive mechanisms of decision making in joint action: A human-robot interaction study," Human movement science, vol. 30, no. 5, pp. 846-868, 2011.

[32] W. Erlhagen, "Internal models for visual perception," Biological cybernetics, vol. 88, no. 5, pp. 409-417, 2003.

[33] C. R. Laing, W. C. Troy, B. Gutkin, and G. B. Ermentrout, "Multiple bumps in a neuronal model of working memory," SIAM Journal on Applied Mathematics, vol. 63, no. 1, pp. 62-97, 2002.

[34] F. Ferreira, W. Erlhagen, and E. Bicho, "Multi-bump solutions in a neural field model with external inputs," Physica D: Nonlinear Phenomena, vol. 326, pp. 32-51, 2016.

[35] S. Coombes and M. Owen, "Exotic dynamics in a firing rate model of neural tissue," in Fluids and Waves: Recent Trends in Applied Analysis: Research Conference, May 11-13, 2006, the Universtiy of Memphis, Memphis, TN, vol. 440. American Mathematical Soc., 2007, p. 123.

[36] G. Houghton, "The problem of serial order: A neural network model of sequence learning and recall," in Current research in natural language generation. Academic Press Professional, Inc., 1990, pp. 287-319.

[37] G. Bradski, G. A. Carpenter, and S. Grossberg, "Store working memory networks for storage and recall of arbitrary temporal sequences," Biological Cybernetics, vol. 71, no. 6, pp. 469-480, 1994

[38] M. R. Silver, S. Grossberg, D. Bullock, M. H. Histed, and E. K. Miller, "A neural model of sequential movement planning and control of eye movements: Item-order-rank working memory and saccade selection by the supplementary eye fields," Neural Networks, vol. 26, pp. 29-58, 2012.

[39] Y. Sandamirskaya and G. Schöner, "An embodied account of serial order: How instabilities drive sequence generation," Neural Networks, vol. 23 , no. 10 , pp. 1164-1179, 2010.

[40] C. Faubel and G. Schöner, "Learning to recognize objects on the fly: a neurally based dynamic field approach," Neural networks, vol. 21, no. 4, pp. 562-576, 2008.

[41] P. M. Lima and W. Erlhagen, "Numerical simulations of twodimensional neural fields with applications to working memory," in 2018 European Control Conference (ECC). IEEE, 2018, pp. 2040-2045.

[42] S. Song, J. H. Howard, and D. V. Howard, "Perceptual sequence learning in a serial reaction time task," Experimental Brain Research, vol. 189, no. 2, pp. $145-158,2008$.

[43] C. Summerfield and F. P. De Lange, "Expectation in perceptual decision making: neural and computational mechanisms," Nature Reviews Neuroscience, vol. 15 , no. 11, p. 745, 2014

[44] C. R. Luo and A. Caramazza, "Temporal and spatial repetition blindness: Effects of presentation mode and repetition lag on the perception of repeated items." Journal of Experimental Psychology: Human Perception and Performance, vol. 22, no. 1, p. 95, 1996.

[45] A. C. Nobre, A. Correa, and J. T. Coull, "The hazards of time," Current opinion in neurobiology, vol. 17, no. 4, pp. 465-470, 2007.

[46] W. Wojtak, F. Ferreira, W. Erlhagen, and E. Bicho, "Learning joint representations for order and timing of perceptual-motor sequences: a dynamic neural field approach," in 2015 International Joint Conference on Neural Networks (IJCNN). IEEE, 2015, pp. 1-7.

[47] R. N. Henson, "Short-term memory for serial order: The start-end model," Cognitive psychology, vol. 36, no. 2, pp. 73-137, 1998.

[48] A. Machado, M. T. Malheiro, and W. Erlhagen, "Learning to time: A perspective," Journal of the Experimental Analysis of Behavior, vol. 92, no. 3, pp. 423-458, 2009.

[49] M. D. Mauk and D. V. Buonomano, "The neural basis of temporal processing," Аnпu. Rev. Neurosci., vol. 27, pp. 307-340, 2004.

[50] J. Gibbon, C. Malapani, C. L. Dale, and C. R. Gallistel, "Toward a neurobiology of temporal cognition: advances and challenges," Current opinion in neurobiology, vol. 7, no. 2, pp. 170-184, 1997.
[51] P. A. Lewis and R. C. Miall, "The precision of temporal judgement: milliseconds, many minutes, and beyond," Philosophical Transactions of the Royal Society B: Biological Sciences, vol. 364, no. 1525, pp. 1897-1905, 2009.

[52] R. Laje, K. Cheng, and D. V. Buonomano, "Learning of temporal motor patterns: an analysis of continuous versus reset timing," Front Integr Neurosci, vol. 5, p. 61, 2011.

[53] J. J. Paton and D. V. Buonomano, "The neural basis of timing: distributed mechanisms for diverse functions," Neuron, vol. 98, no. 4, pp. 687-705, 2018.

[54] J. Reutimann, V. Yakovlev, S. Fusi, and W. Senn, "Climbing neuronal activity as an event-based cortical representation of time," Journal of Neuroscience, vol. 24, no. 13, pp. 3295-3303, 2004.

[55] D. Durstewitz, "Self-organizing neural integrator predicts interval times through climbing activity," Journal of Neuroscience, vol. 23, no. 12, pp. $5342-5353,2003$

[56] A. Cleeremans and J. L. McClelland, "Learning the structure of event sequences." Journal of Experimental Psychology: General, vol. 120, no. 3, p. 235,1991

[57] Z. C. Lipton, J. Berkowitz, and C. Elkan, "A critical review of recurrent neural networks for sequence learning," arXiv preprint arXiv:1506.00019, 2015.

[58] R. Laje and D. V. Buonomano, "Robust timing and motor patterns by taming chaos in recurrent neural networks," Nature neuroscience, vol. 16 , no. 7 , p. $925,2013$.

[59] E. Sousa, W. Erlhagen, and E. Bicho, "On observational learning of hierarchies in sequential tasks: A dynamic neural field model," in Computational Models of Cognitive Processes. World Scientifc, Jan. 2014, pp. 196-210. 